\title{
Transsacral Screw Safe Zone Size by Sacral Segmentation Variations
}

\author{
John J. Lee, ${ }^{1}$ Samuel L. Rosenbaum, ${ }^{1}$ Alex Martusiewicz, ${ }^{1}$ Sven A. Holcombe, ${ }^{2}$ Stewart C. Wang, ${ }^{2}$ James A. Goulet ${ }^{1}$ \\ ${ }^{1}$ Department of Orthopaedic Surgery, University of Michigan, 1500 E Medical Center Dr, 2912 Taubmann Center, SPC 5328, Ann Arbor 48109, \\ Michigan, ${ }^{2}$ Department of General Surgery, University of Michigan, Ann Arbor, Michigan \\ Received 30 June 2014; accepted 25 August 2014 \\ Published online 17 September 2014 in Wiley Online Library (wileyonlinelibrary.com). DOI 10.1002/jor.22739 \\ ABSTRACT: Variations in sacral segmentation may preclude safe placement of transsacral screws for posterior pelvis fixation. We \\ developed a novel automated 3D technique to determine the safe zone size for transsacral screws in the upper two sacral segments in \\ 526 adult pelvis computed tomography scans. Safe zone sizes were then compared by gender and sacral segmentation variations \\ (number of neuroforamen and the presence/absence of lumbosacral transitional vertebrae, \pm LSTV). Ten millimeters was used as the \\ safety threshold for a large screw. $3(0.6 \%), 366(70 \%)$, and $157(30 \%)$ sacra had 3, 4, or 5 neuroforamen, respectively. Eighty-eight \\ $(17 \%)$ were +LSTV. Safe zone size depended on gender, number of neuroforamen in -LSTV sacra and presence of LSTV $(p<0.001)$ but \\ not on the uni- or bilateral nature of the LSTV. 17\% of -LSTV sacra were below the safety threshold in S1, 27\% in S2, whereas 3\% of \\ + LSTV sacra were below in S1, 74\% in S2. Of - LSTV sacra that cannot take an S1 screw safely, $77 \%$ can do so in S2, leaving only $4 \%$ \\ of sacra that cannot accommodate a screw safely in either upper segment. The results demonstrate a predictable pattern of safe zone \\ size based on gender and sacral segmentation variations. () 2014 Orthopaedic Research Society. Published by Wiley Periodicals, Inc. J \\ Orthop Res 33:277-282, 2015.
}

Keywords: lumbosacral transitional vertebrae; sacral dysmorphism; sacral safe zone; sacral segmentation; transsacral screw

Transsacral screws have become a popular method of fixation of the posterior pelvis as they can be placed percutaneously, avoiding posterior dissection and potential wound complications, with minimal blood loss and with the patient supine or prone. ${ }^{1,2}$ Transsacral screws are technically challenging in that they span both foraminal regions of the sacrum within an intraosseous corridor that is dependent on sacral morphology. ${ }^{3-12}$ With advances in the technique of fluoroscopically guided percutaneous screw placement and anatomic understanding of the sacrum and surrounding neurovascular structures, there has been a low incidence of neurovascular injury. ${ }^{3,13-17}$

The lumbosacral junction is the most variable portion of the spine with variations in sacral segmentation, thought to be related to its load-related fusion, seen in both number of segments, typically four to six, and lumbosacral transitional vertebrae (LSTV) defined as either sacralization of the lowest lumbar segment or lumbarization of the upper sacral segment. ${ }^{18-22}$ LSTV are estimated to be present in $4-30 \%$ of the population and have been described since at least the early 20th century. ${ }^{23-25}$ Castellvi et al. identified four types based on morphology in their classification system for LSTV. $^{24,25}$ Type I includes unilateral (Ia) or bilateral (Ib) dysplastic transverse processes; type II, incomplete unilateral (IIa) or bilateral (IIb) lumbarization/sacralization with an enlarged transverse process that has a diarthrodial joint between itself and the sacrum; type III, unilateral (IIIa) or bilateral (IIIb) lumbarization/ sacralization with complete osseous fusion of the transverse process(es) to the sacrum; and type IV,

Conflicts of interest: No external funding was used for this study. The authors have no relevant professional and financial affiliations to disclose.

Correspondence to: John J. Lee (T: 734-936-5780;

F: 734-615-8568; E-mail: jleecal@hotmail.com)

(C) 2014 Orthopaedic Research Society. Published by Wiley Periodicals, Inc. unilateral type II transition with a type III on the contralateral side. ${ }^{21,22}$ While useful for describing the relationship of the transitional segment to that below it, this system does not provide information on whether the transitional segment is a lumbarized sacral element or a sacralized lumbar element given the significant difficulty in determining this on imaging alone. ${ }^{24}$

Studies to date regarding sacral morphology and its correlation with the size of the safe zone for iliosacral and transsacral screws have primarily focused on whether the sacrum was normal or dysmorphic as described by Routt et al. ${ }^{3,6,7,11,14}$ However, it is unclear as to how many of the dysmorphic features described by Routt et al. are needed to label a sacrum as such nor which features are more specific for it. ${ }^{3}$ It is likely that what is described as dysmorphic is a variation of Castellvi LSTV types IIb, IIIb, and IV. ${ }^{25}$ In the largest study to date, Gardner et al. measured the transverse safe zone in normal and dysmorphic sacra in both upper segments in 50 patients and found mean widths of 12.9 and $1.2 \mathrm{~mm}$ for the upper sacral segment, and 10.3 and $14.0 \mathrm{~mm}$ for the second sacral segment for 26 normal and 24 dysmorphic sacra, respectively. ${ }^{7}$ The safe zone size of the upper sacral segments has yet to be correlated to variations in sacral segmentation.

The purpose of this study was to quantify the size of the safe zone for transsacral screws in the upper (S1) and second (S2) sacral segments and determine if this is dependent on gender and patterns of sacral segmentation. Our hypothesis was based on the load-related fusion concept of the lumbosacral junction, specifically that an increase in the number of neuroforamen shifts the area of load distribution of the sacroiliac joint inferiorly thus diminishing the safe zone size for S1 and increasing it for S2 and similarly, the presence of an LSTV shifts the area of load distribution of the sacroiliac joint superiorly thus increasing the safe zone size for S1 and diminishing it for $\mathrm{S} 2 .{ }^{19-22}$ 


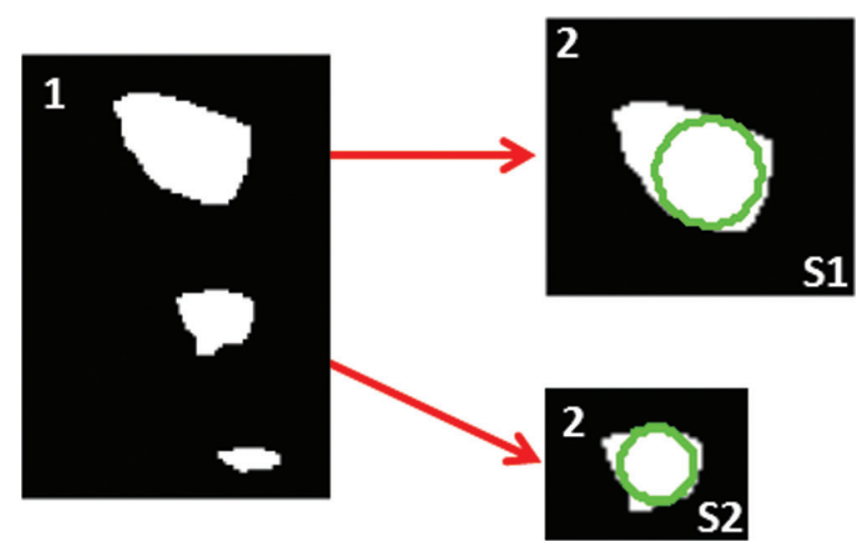

Figure 1. Upper and second sacral segment safe zones as seen on the sagittal (1) with maximum diameter circle fit (2).

\section{METHODS}

Five hundred twenty-six adult (18 years and older) pelvis computed tomography (CT) scans without bilateral sacral fractures, that had been uploaded from our clinical PACS system to our institution's research archive and analysis database, were analyzed with a novel automated MATLAB script (Mathworks, Natwick, MA) that algorithmically identified bony landmarks (anterior superior and inferior iliac spines, sacral midline, and anterior sacral foramina points) that were used to rotate pelves in the axial and coronal planes to create a reconstruction plane that was orthonormal to the true sagittal plane. If a unilateral displaced fracture was present, the uninjured hemipelvis was analyzed. The maximum diameter of an intraosseous cylinder traversing the sacrum orthogonal to the sagittal reconstruction plane,
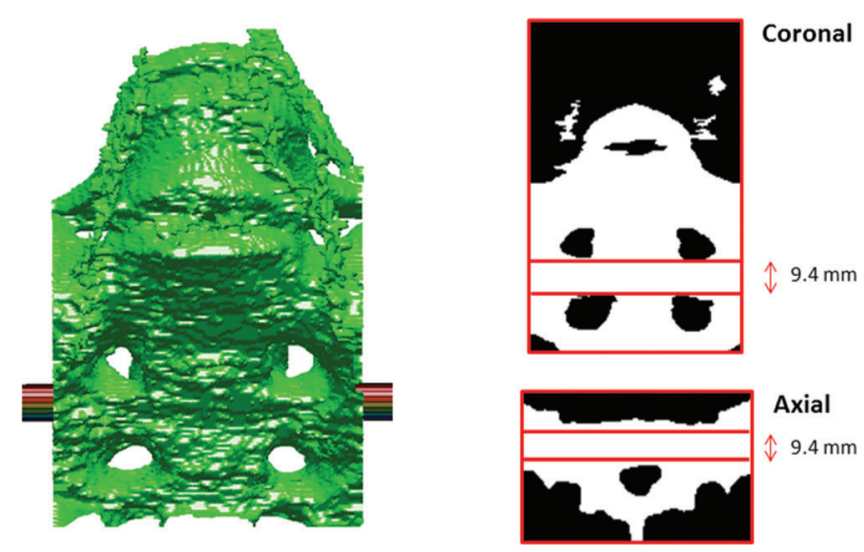

Figure 2. Safe zone diameter confirmation images at the second sacral segment. Images were reviewed for all sacra for accuracy of the automated safe zone size determination.

representing the trajectory for maximal safe zone size of a transsacral screw, was automatically determined for S1 and S2 (Fig. 1). These were verified manually for accuracy (Fig. 2). Because identifying an LSTV as either a lumbarized sacral element or a sacralized lumbar element can be problematic, ${ }^{24}$ we chose to define the upper sacral segment (S1) as the sacral segment below an LSTV or the most inferior lumbar vertebrae. This is in contradistinction to prior studies on safe zone size in dysmorphic sacra, which presumably defined the LSTV as the upper sacral segment. 6,7

Sacra were characterized by the larger of the number of anterior neuroforamen present on either side, the presence or absence of LSTV ( \pm LSTV), and whether the LSTV was uni- or bilateral (Fig. 3). ${ }^{24}$ The number of anterior neuroforamen included the foramen between the LSTV and S1. LSTV

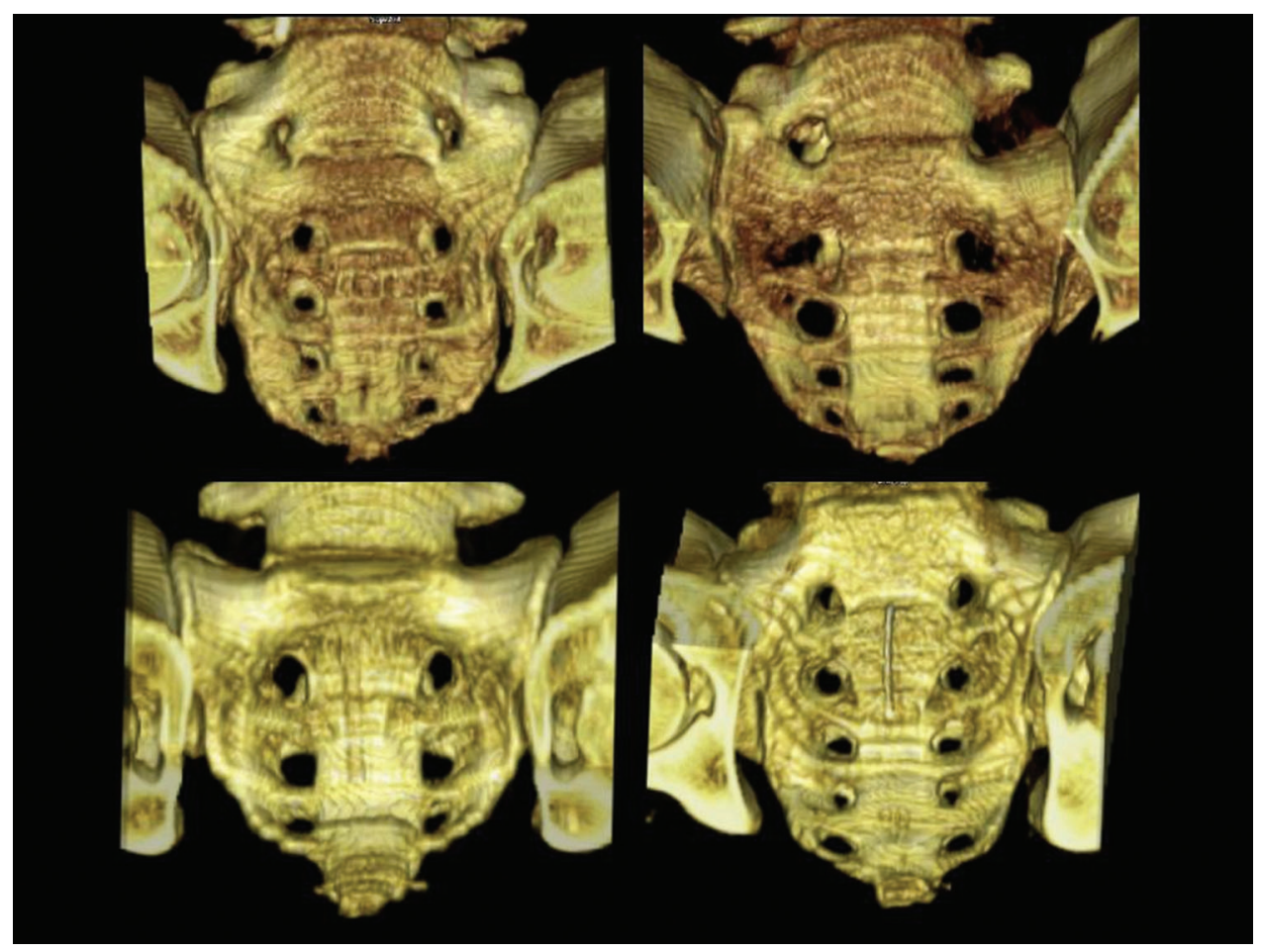

Figure 3. Bilateral (top left) and unilateral (top right) lumbosacral transitional vertebrae, sacra with three (bottom left) and five (bottom right) anterior neuroforamen. 
were identified as such per the description for Castellvi types II and higher. Castellvi types I, described as having unilateral or bilateral dysplastic transverse processes from the LSTV without an articulation with the sacrum, were thought to be less objective in our analysis. ${ }^{25}$

The significance of associations between the safe zone size of the upper two sacral segments and gender, number of neuroforamen, the presence or absence of LSTV, and whether the LSTV was unilateral or bilateral were determined with one-way ANOVA or two-tailed paired $t$ tests with the assumption of unequal variances. A $p$-value of less than 0.05 was considered significant.

\section{RESULTS}

Data are presented in mean (standard deviation) or in count (proportion, with or without 95\% confidence interval). Of the 526 pelves, 305 (58\%) were male and $221(42 \%)$ female. Average age was 34.8 (15.9) years with a range of $18-106$ years. $3(0.6 \%)$ sacra had three neuroforamen, $366(70 \%)$ had four, and $157(30 \%)$ had five (Fig. 3). $88(17 \%, 14-20 \%)$ pelves, including 30 (34\%) females and $58(66 \%)$ males, were identified as having a lumbosacral transitional vertebrae (+LSTV). Of the +LSTV sacra, $13(15 \%, 7-22 \%)$ had four neuroforamen, 75 (85\%, 78-93\%) had five neuroforamen, 47 (53\%, 43-64\%) were bilateral and $41(47 \%, 36-57 \%)$ were unilateral (21 left, 20 right).

Safe zone size was dependent on gender $(p<0.001)$, number of neuroforamen in -LSTV sacra $(p<0.001)$ and presence or absence of LSTV $(p<0.001)$ but was not dependent on whether the LSTV was unilateral or bilateral (Table 1). In -LSTV sacra, males had $10 \%$ larger S1 and 20\% larger S2 safe zones than females $(p=0.0001$ for S1, $p<0.0001$ for S2). In +LSTV sacra, males had $12 \%$ larger S1 and 7\% larger S2 safe zones than females ( $p=0.013$ for S1 only). - LSTV sacra with four neuroforamen had a 27\% larger safe zone in S1 $(p<0.0001)$ and a $10 \%$ smaller safe zone in $\mathrm{S} 2(p=$ 0.0007 ) than those with five neuroforamen (Table 2). Safe zone size in +LSTV sacra did not depend on the number of neuroforamen. Safe zone size in +LSTV sacra was $23 \%$ larger in S1 and $44 \%$ smaller in S2 than in -LSTV sacra $(p<0.0001$ for both S1 and S2).

Using a safety threshold of $10 \mathrm{~mm}$ for placement of a large screw, ${ }^{7} 83 \%(80-87 \%)$ of -LSTV sacra were above the threshold in S1 and 73\% (69-77\%) in S2 whereas in +LSTV sacra 97\% (93-100\%) were above the safety threshold in S1 and 26\% (17-35\%) in S2 (Table 2). Of the $17 \%$ of -LSTV sacra that cannot take an S1 screw safely, 77\% (67-87\%) can safely take an S2 screw, leaving $4 \%(2-6 \%)$ of - LSTV sacra that are below the safety threshold in both $\mathrm{S} 1$ and $\mathrm{S} 2.4 \%$ (2$5 \%$ ) of -LSTV sacra can safely take two large cannulated screws in S1 (using a safety threshold of $20 \mathrm{~mm}$ or $10 \mathrm{~mm} / \mathrm{screw})$ while $22 \%(13-30 \%)$ of +LSTV sacra could take two screws. Second sacral segments in either group cannot accept two large screws. In -LSTV sacra, $60 \%(49-70 \%)$ with five neuroforamen and $89 \%$ (85-92\%) of sacra with four neuroforamen were above the safety threshold in S1 while $74 \%$ (65-84\%) with
Table 1. Sacral Segment Safe Zone Mean Diameters (Standard Deviation) by Gender, Number of Sacral Foramen, Uni/Bilateral Transitional Vertebrae, and the Presence or Absence of Transitional Vertebrae ( \pm LSTV)

\begin{tabular}{|c|c|c|c|}
\hline & $+\mathrm{LSTV}$ & -LSTV & $+/-\mathrm{LSTV}$ \\
\hline \multicolumn{4}{|l|}{ Male } \\
\hline S1 & $17.7(3.6)$ & $14.5(3.8)$ & $15.1(3.9)$ \\
\hline $\mathrm{S} 2$ & $8.2(2.9)$ & $12.4(2.9)$ & $11.6(3.4)$ \\
\hline \multicolumn{4}{|c|}{ Female } \\
\hline $\mathrm{S} 1$ & $15.8(2.8)$ & $13.2(3.5)$ & $13.5(3.5)$ \\
\hline $\mathrm{S} 2$ & $7.6(2.4)$ & $10.3(2.7)$ & $10.0(2.8)$ \\
\hline \multicolumn{4}{|c|}{ Male and female } \\
\hline $\mathrm{S} 1$ & $17.1(3.5)$ & $13.9(3.7)$ & $14.4(3.8)$ \\
\hline $\mathrm{S} 2$ & $8.0(2.8)$ & $11.5(3.0)$ & $10.9(3.3)$ \\
\hline \multicolumn{4}{|c|}{ Three } \\
\hline $\mathrm{S} 1$ & None & $15.3(1.3)$ & $15.3(1.3)$ \\
\hline $\mathrm{S} 2$ & None & $7.4(2.5)$ & $7.4(2.5)$ \\
\hline \multicolumn{4}{|l|}{ Four } \\
\hline $\mathrm{S} 1$ & $16.1(4.4)$ & $14.5(3.4)$ & $14.5(3.4)$ \\
\hline $\mathrm{S} 2$ & $6.9(1.8)$ & $11.3(2.9)$ & $11.2(3.0)$ \\
\hline \multicolumn{4}{|l|}{ Five } \\
\hline $\mathrm{S} 1$ & $17.2(3.3)$ & $11.3(4.0)$ & $14.2(4.7)$ \\
\hline $\mathrm{S} 2$ & $8.2(2.9)$ & $12.6(3.3)$ & $10.5(3.8)$ \\
\hline \multicolumn{4}{|c|}{ Unilateral } \\
\hline $\mathrm{S} 1$ & $17.3(3.7)$ & NA & $17.3(3.7)$ \\
\hline $\mathrm{S} 2$ & $8.3(2.6)$ & NA & $8.3(2.6)$ \\
\hline \multicolumn{4}{|c|}{ Bilateral } \\
\hline $\mathrm{S} 1$ & $16.8(3.3)$ & NA & $16.8(3.3)$ \\
\hline $\mathrm{S} 2$ & $7.7(2.9)$ & NA & $7.7(2.9)$ \\
\hline
\end{tabular}

Safe zone size was dependent on gender, the number of neuroforamen in-LSTV sacra and the presence or absence of LSTV (all $p<0.001$ ) but was not dependent on whether the LSTV was unilateral or bilateral. S1, upper sacral segment; S2, second sacral segment.

five neuroforamen and 73\% (68-78\%) with four neuroforamen were above in the second sacral segment.

\section{DISCUSSION}

To avoid injury to nearby neurovascular structures that come in close proximity to the cortical borders of the sacrum, transsacral screws must traverse sacra intraosseously in a transverse or orthogonal trajectory to the sagittal plane through bilateral neuroforaminal regions, anterior to the sacral canal at midline, with minor deviations from this trajectory diminishing the effective safe zone size. ${ }^{3,10,26,27}$ In this study, we present a novel method of defining and grouping variations of sacral segmentation using number of neuroforamen, which may be a surrogate for number of sacral elements, and the presence or absence of an LSTV for purposes of predicting safe zone size for the upper two sacral segments utilizing the load-related fusion concept of the lumbosacral junction. ${ }^{19-22}$ Other novel findings in this study are: (1) the safe zone size for the upper sacral segments with unilateral LSTV are similar to that with bilateral LSTV; and (2) there is large variability in the size of the LSTV transverse 
Table 2. Sacral Segment Safe Zones Meeting Safety Threshold (95\% Confidence Interval) by Gender, Number of Sacral Foramen, Uni/Bilateral Transitional Vertebrae and the Presence or Absence of Transitional Vertebrae ( \pm LSTV)

\begin{tabular}{|c|c|c|c|}
\hline & +LSTV & -LSTV & +/-LSTV \\
\hline \multicolumn{4}{|l|}{ Male } \\
\hline $\mathrm{S} 1$ & $97 \%(92-100 \%)$ & $85 \%(81-90 \%)$ & $88 \%(84-91 \%)$ \\
\hline $\mathrm{S} 2$ & $31 \%(19-43 \%)$ & $84 \%(79-88 \%)$ & $74 \%(69-79 \%)$ \\
\hline \multicolumn{4}{|c|}{ Female } \\
\hline $\mathrm{S} 1$ & $97 \%(90-100 \%)$ & $80 \%(74-86 \%)$ & $82 \%(77-87 \%)$ \\
\hline $\mathrm{S} 2$ & $17 \%(3-30 \%)$ & $59 \%(52-66 \%)$ & $53 \%(47-60 \%)$ \\
\hline \multicolumn{4}{|c|}{ Male and female } \\
\hline $\mathrm{S} 1$ & $97 \%(93-100 \%)$ & $83 \%(80-87 \%)$ & $85 \%(82-88 \%)$ \\
\hline $\mathrm{S} 2$ & $26 \%(17-35 \%)$ & $73 \%(69-77 \%)$ & $65 \%(61-69 \%)$ \\
\hline \multicolumn{4}{|c|}{ Three } \\
\hline $\mathrm{S} 1$ & None & $100 \%{ }^{\mathrm{a}}$ & $100 \%^{\mathrm{a}}$ \\
\hline $\mathrm{S} 2$ & None & $33 \%^{\mathrm{a}}$ & $33 \%^{\mathrm{a}}$ \\
\hline \multicolumn{4}{|l|}{ Four } \\
\hline $\mathrm{S} 1$ & $92 \%(78-100 \%)$ & $89 \%(85-92 \%)$ & $89 \%(86-82 \%)$ \\
\hline $\mathrm{S} 2$ & $0 \%$ & $73 \%(68-78 \%)$ & $70 \%(66-75 \%)$ \\
\hline \multicolumn{4}{|l|}{ Five } \\
\hline $\mathrm{S} 1$ & $97 \%(94-100 \%)$ & $60 \%(49-70 \%)$ & $77 \%(70-84 \%)$ \\
\hline $\mathrm{S} 2$ & $31 \%(20-41 \%)$ & $74 \%(65-84 \%)$ & $54 \%(46-61 \%)$ \\
\hline \multicolumn{4}{|c|}{ Unilateral } \\
\hline $\mathrm{S} 1$ & $95 \%(89-100 \%)$ & NA & $95 \%(89-100 \%)$ \\
\hline $\mathrm{S} 2$ & $26 \%(15-43 \%)$ & NA & $26 \%(15-43 \%)$ \\
\hline \multicolumn{4}{|c|}{ Bilateral } \\
\hline $\mathrm{S} 1$ & $98 \%(94-100 \%)$ & NA & $98 \%(94-100 \%)$ \\
\hline $\mathrm{S} 2$ & $20 \%(11-36 \%)$ & NA & $20 \%(11-36 \%)$ \\
\hline
\end{tabular}

${ }^{\mathrm{a}}$ Confidence intervals not provided given $n=3$.

processes, some of which can accept a large transverse screw. Furthermore, this is the largest study to date reporting safe zone sizes for transsacral screws, which were determined systematically utilizing a novel highthroughput three-dimensional analysis of the sacrum rather than a single CT slice. ${ }^{6,7}$

The number of neuroforamen had a significant association with safe zone size in both upper sacral segments with S1 having a negative association with and S2 a positive association with number of neuroforamen (Tables 1 and 2). The presence of five neuroforamen could indicate the existence of either: (1) sacralization of a lower lumbar vertebrae; (2) six sacral segments; or (3) lateral fusion of the lower sacral segment with the coccyx establishing a foramen. While the majority of our sacra had four neuroforamen (366/ $526,70 \%$ ), there were a large number of sacra with five neuroforamen $(157 / 526,30 \%)$ with half of these having an LSTV (75/157, 48\%). We also identified three sacra with three neuroforamen which could indicate the existence of either: (1) a completely lumbarized sacral segment; (2) four sacral segments; or (3) an incompletely fused lower sacral segment in a five segment sacrum. The association of safe zone size with number of neuroforamen can be explained by the load-fusion relationship of sacral segmentation, with larger sacra (five neuroforamen) distributing more axial load evenly between the upper two sacral segments while smaller sacra (three or four neuroforamen) distribute more through an S1 with a larger articular surface with the ilium. ${ }^{21,22}$

LSTV are common and likely represent the majority of variation at the lumbosacral junction with a reported prevalence of $4-30 \%$ (17\% in our study) and demonstrate varying morphology from unilateral or bilateral diarthrodial joints and/or complete fusion with the sacrum. ${ }^{24}$ LSTV have a distinctive appearance with a large residual disk space and oftentimes a distinct anterior convexity between the LSTV and the sacrum inferiorly on midline sagittal imaging in contrast to the anterior concavity noted throughout sacra without an LSTV. This morphology oftentimes results in an acute alar slope as described by Routt et al. ${ }^{3}$ with an anterior neuroforamen located posteriorly and coursing over the convex ala of the upper sacral segment (Fig. 3). Determining whether the LSTV is a lumbarized sacral element or a sacralized lumbar element is problematic, especially given the variability above and below where the thoracolumbar spine can vary in the number of vertebrae and sacra can have anywhere from four to six segments. $^{24}$ The presence of an LSTV indicates a capacious S1 safe zone that can nearly universally accept a large screw (97\%) and a relatively small S2 safe zone, of which only a quarter $(26 \%)$ can accept a large screw safely (Table 2). Whether the LSTV was unilateral or bilateral had no effect on the safe zone size of the upper two segments. Although 30 of the 47 bilateral LSTV in our study had no measurable transverse safe zones due to geometric impossibility, 5 of the 17 bilateral LSTV that had a measurable transverse safe zone had an LSTV safe zone size greater than $10 \mathrm{~mm}$, with the mean diameter of these 17 being 8.4 (5.1) $\mathrm{mm}$ (Fig. 4). This is in contrast to a series reported by Gardner et al. where a $10 \mathrm{~mm}$ safe zone was not possible in 24 dysmorphic sacra. ${ }^{7}$ This is likely due to differences in the dichotomization of sacra in their series by dysmorphism ${ }^{3}$ and by the presence or absence of LSTV in our study. Our results demonstrate the large variability of the size of the LSTV transverse processes as noted by Gardner et al. ${ }^{7}$ and support the load-fusion concept of sacral segmentation. ${ }^{19-22}$ Sacra with large LSTV safe zones $(>10 \mathrm{~mm})$ had smaller S1 $(15.7$ vs. $17.3 \mathrm{~mm})$ and $\mathrm{S} 2(6.2$ vs. $8.5 \mathrm{~mm})$ safe zones than sacra with LSTV that had smaller or no safe zones although no statistical analysis was performed due to the small sample size.

Safe zone size analyses to date have primarily focused on an oblique safe zone corridor pertinent to unilateral iliosacral screws in the upper sacral segment and have reported the safe zone size in the second sacral segment in dysmorphic sacra to be capacious. ${ }^{6,9,11,12,27}$ Conflitti et al. manually measured on their PACS the safe zone for unilateral iliosacral screws in 24 dysmorphic sacra and found the average width of the first sacral segment to be $13.2 \mathrm{~mm}$ and 


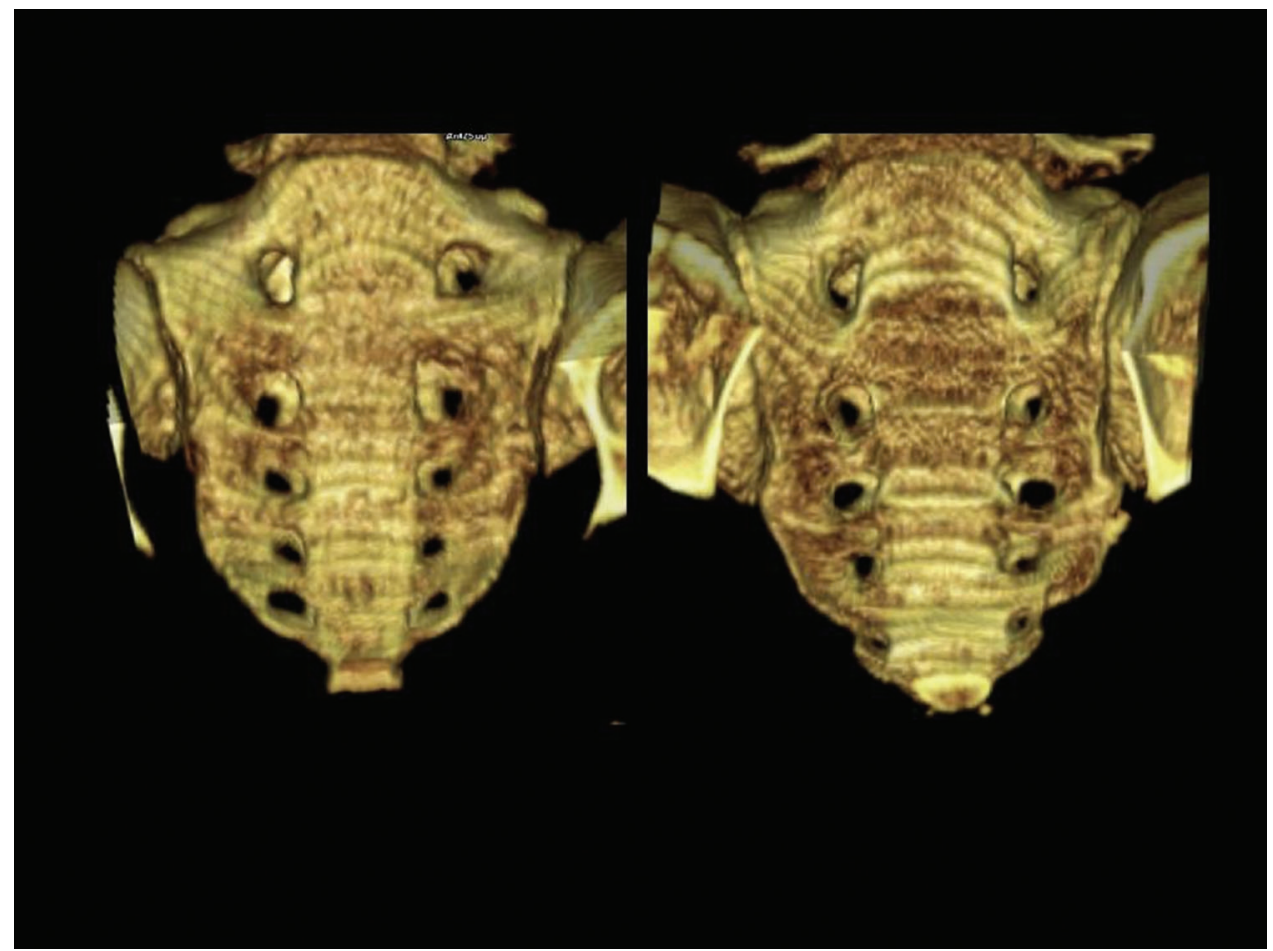

Figure 4. Sacra with bilateral lumbosacral transitional vertebrae that cannot (left) and can (right) safely accept a transsacral screw across the transitional vertebrae using a $10 \mathrm{~mm}$ safety threshold. Note the more acute alar slope on the sacrum that cannot accept the screw safely.

the second sacral segment to be $15.2 \mathrm{~mm}$, noting that in the second sacral segment iliosacral screws could be placed transversely to end in the contralateral ilium whereas they had to be placed obliquely in the first segment. ${ }^{6}$ Gardner et al. measured the safe zone width for a transsacral screw on CT simulated inlet and outlet views in 50 patients, of which 22 were dysmorphic, and found mean widths of 12.9 (3.7) $\mathrm{mm}$ and 1.2 (6.6) $\mathrm{mm}$ for the upper sacral segment and 10.3 (2.3) $\mathrm{mm}$ and 14.0 (1.9) $\mathrm{mm}$ for the second sacral segment for normal and dysmorphic sacra, respectively. ${ }^{7}$ These results are similar to ours when acknowledging the slightly different definitions used for the upper sacral segment where the LSTV was labeled as the upper sacral segment in prior studies but labeled as a distinct LSTV level with the upper sacral segment below it in our study. We defined S1 as the segment just below the LSTV for four reasons: (1) it is difficult to establish whether an LSTV is a lumbarized sacral segment or a sacralized lumbar segment; (2) LSTV morphology is variable and they may or may not be fused with the sacrum; (3) the majority of LSTV do not have a transverse safe zone; and (4) LSTV can be unilateral $(41 / 88$ or $47 \%$ in this study) or bilateral, with the size of the safe zones similar between the two groups. $^{24,25}$ Dysmorphic sacra in prior studies likely included Castellvi LSTV types IIb, IIIb, or IV in addition to other sacra fulfilling the subjective criteria for dysmorphism thus possibly accounting for a higher prevalence of dysmorphic sacra in prior studies when compared to the prevalence of +LSTV sacra in this study, although this could be due to sample size variances alone as the reported prevalence varies widely for both LSTV and dysmorphic sacra. ${ }^{5-7,24,25,28}$

Strengths of this study include a large sample size, an arguably more objective and simpler method of classifying sacra by number of anterior neuroforamen and the presence or absence of an LSTV in contrast to using a constellation of dysmorphic features, and a systematic three-dimensional automated technique to measure transsacral screw safe zone size. Primary limitations of this study involve our automated image processing technique, the accuracy of segmenting the sacrum from surrounding tissue with grayscale thresholding and other morphological operators, and our method of determining the cardinal axes of the pelvis with an algorithmic anatomical point detection technique. It is important to note that safe zone size determinations include the cortical bone rim, which may give a slightly overestimated or a maximum limit for safe zone size. Furthermore, an arbitrary safety threshold of $10 \mathrm{~mm}$ for safe placement of a large screw $(6.5-8.0 \mathrm{~mm})$ as used prior may be generous. ${ }^{7}$ While our study demonstrated that no second sacral segment could accept two transsacral screws, placement of two screws in this segment has been reported. ${ }^{8}$

The load-related fusion theory for sacral segmentation variations explains well the correlation of anterior neuroforamen and presence of LSTV to transsacral screw safe zone size. The presence of LSTV, whether 
unilateral or bilateral, nearly universally indicates an upper sacral segment that is large enough for safe screw placement. The second sacral segment is often a safe alternative to transsacral screw placement if the upper segment safe zone is too small. Only $4 \%$ of sacra cannot accept a large screw in either upper segment. A small proportion of LSTV with especially large transverse processes may be able to accommodate a transsacral screw. Given the prevalence of sacral segmentation variations, the wide variation of LSTV morphology and fusion patterns with the sacrum, and with a comprehensive understanding of the safe zone, future research may want to consider the effect of these parameters and the load-related fusion concept on posterior pelvis fixation stability.

\section{REFERENCES}

1. Routt ML Jr, Simonian PT. 1996. Closed reduction and percutaneous skeletal fixation of sacral fractures. Clin Orthop Relat Res 329:121-128.

2. Gardner MJ, Chip Routt ML Jr. 2010. The antishock iliosacral screw. J Orthop Trauma 24:e86-e89.

3. Routt ML Jr, Simonian PT, Agnew SG, et al. 1996. Radiographic recognition of the sacral alar slope for optimal placement of iliosacral screws: a cadaveric and clinical study. J Orthop Trauma 10:171-177.

4. Ziran BH, Smith WR, Towers J, et al. 2003. Iliosacral screw fixation of the posterior pelvic ring using local anaesthesia and computerised tomography. J Bone Joint Surg Br 85:411-418.

5. Miller AN, Routt ML Jr. 2012. Variations in sacral morphology and implications for iliosacral screw fixation. J Am Acad Orthop Surg 20:8-16.

6. Conflitti JM, Graves ML, Chip Routt ML Jr. 2010. Radiographic quantification and analysis of dysmorphic upper sacral osseous anatomy and associated iliosacral screw insertions. J Orthop Trauma 24:630-636.

7. Gardner MJ, Morshed S, Nork SE, et al. 2010. Quantification of the upper and second sacral segment safe zones in normal and dysmorphic sacra. J Orthop Trauma 24:622-629.

8. Gardner MJ, Routt ML Jr. 2011. Transiliac-transsacral screws for posterior pelvic stabilization. J Orthop Trauma 25:378-384.

9. Noojin FK, Malkani AL, Haikal L, et al. 2000. Cross-sectional geometry of the sacral ala for safe insertion of iliosacral lag screws: a computed tomography model. J Orthop Trauma 14:31-35.

10. Templeman D, Schmidt A, Freese J, et al. 1996. Proximity of iliosacral screws to neurovascular structures after internal fixation. Clin Orthop Relat Res 329:194-198.
11. Carlson DA, Scheid DK, Maar DC, et al. 2000. Safe placement of S1 and S2 iliosacral screws: the "vestibule" concept. J Orthop Trauma 14:264-269.

12. Arman C, Naderi S, Kiray A, et al. 2009. The human sacrum and safe approaches for screw placement. J Clin Neurosci 16:1046-1049.

13. Nork SE, Jones CB, Harding SP, et al. 2001. Percutaneous stabilization of U-shaped sacral fractures using iliosacral screws: technique and early results. J Orthop Trauma 15: 238-246.

14. Moed BR, Geer BL. 2006. S2 iliosacral screw fixation for disruptions of the posterior pelvic ring: a report of 49 cases. J Orthop Trauma 20:378-383.

15. Routt ML Jr, Simonian PT, Mills WJ. 1997. Iliosacral screw fixation: early complications of the percutaneous technique. J Orthop Trauma 11:584-589.

16. Peng KT, Huang KC, Chen MC, et al. 2006. Percutaneous placement of iliosacral screws for unstable pelvic ring injuries: comparison between one and two C-arm fluoroscopic techniques. J Trauma 60:602-608.

17. Wolinsky P, Lee M. 2007. The effect of C-arm malrotation on iliosacral screw placement. J Orthop Trauma 21:427-434.

18. Gray H, Williams PL, Gray H. 1989. Gray's anatomy. Edinburgh, New York: C. Livingstone.

19. Mahato NK. 2011. Relationship of sacral articular surfaces and gender with occurrence of lumbosacral transitional vertebrae. Spine J 11:961-965.

20. Mahato NK. 2010. Morphological traits in sacra associated with complete and partial lumbarization of first sacral segment. Spine J 10:910-915.

21. Pal G. 1989. Weight transmission through the sacrum in man. J Anat 162:9-17.

22. Pal G, Routal R. 1991. Relationship between the articular surface area of a bone and the magnitude of stress passing through it. Anat Rec 230:570-574.

23. Moore B. 1925. Sacralization of the fifth lumbar vertebra. J Bone Joint Surg Am 7:271-278.

24. Konin GP, Walz DM. 2010. Lumbosacral transitional vertebrae: classification, imaging findings, and clinical relevance. AJNR Am J Neuroradiol 31:1778-1786.

25. Castellvi AE, Goldstein LA, Chan DP. 1984. Lumbosacral transitional vertebrae and their relationship with lumbar extradural defects. Spine (Phila Pa 1976) 9:493-495.

26. Reilly MC, Bono CM, Litkouhi B, et al. 2003. The effect of sacral fracture malreduction on the safe placement of iliosacral screws. J Orthop Trauma 17:88-94.

27. Day CS, Prayson MJ, Shuler TE, et al. 2000. Transsacral versus modified pelvic landmarks for percutaneous iliosacral screw placement-a computed tomographic analysis and cadaveric study. Am J Orthop (Belle Mead NJ) 29:16-21.

28. Mahato NK. 2010. Complete sacralization of L5 vertebrae: traits, dimensions, and load bearing in the involved sacra. Spine J 10:610-615. 